\title{
On resolving the selective interspecific information use vs. owner aggression hypothesis dilemma-a commentary
}

\author{
Jakub Szymkowiak ${ }^{1}$ \\ Received: 11 April 2018 / Revised: 12 April 2019 / Accepted: 7 May 2019 / Published online: 20 May 2019 \\ (C) The Author(s) 2019
}

\begin{abstract}
Using information acquired from other individuals (social information use) is ubiquitous in animals and not restricted to withinspecies context. Some of the textbook examples of interspecific information use in the wild has been provided using apparent novel niche experiment approach, with migratory flycatcher species (Ficedula spp.) cueing on apparent nest site preferences (geometric symbols) and clutch sizes of resident great tits (Parus major) and blue tits (Cyanistes caeruleus) to choose their own nest site. However, the interpretation of these results has recently been challenged. It has been argued that there is no evidence that flycatchers visit tit nests to collect information about clutch size or that flycatchers are capable of "counting" tit eggs. Instead, it has been proposed that the decision-making patterns of flycatchers found in earlier studies resulted from aggressive interactions, not information use, between tits and flycatchers (termed owner aggression hypothesis). Here, I contribute to the debate around both hypotheses and specifically explore the remaining points of disagreement, related to the timing of flycatchers prospecting and the cognitive abilities needed to assess tit clutch size. I argue how interannual variation in phenology can explain the apparent discrepancy between the observed and predicted timing of flycatchers prospecting. I also argue why assessing tit clutch size neither requires "counting" eggs nor any sophisticated cognitive capacities. Finally, I propose experimental designs to test the hypotheses on the underlying mechanisms explaining the observed patterns, which will allow taking further leaps in resolving the selective interspecific information use vs. owner aggression hypothesis dilemma.
\end{abstract}

Keywords Cavity nesters $\cdot$ Ficedula $\cdot$ Habitat selection $\cdot$ Paridae $\cdot$ Social information

\section{Introduction}

Social information use, when individuals use information acquired from other individuals, is ubiquitous in the animal kingdom. A wide range of animals from insects to humans rely on social cues when deciding, e.g., with whom to mate, where to breed or rest, what and where to feed, or when and from whom to escape (Seppänen et al. 2007; Hoppit and Laland 2013). This phenomenon not only is restricted to within-species context but also operates across species boundaries (Avarguès-Weber et al. 2013). For example, many animals use the presence of ecologically similar heterospecifics as a cue for settlement decisions (e.g., Thomson et al. 2003;

Jakub Szymkowiak

jszym@amu.edu.pl

1 Population Ecology Lab, Institute of Environmental Biology, Faculty of Biology, Adam Mickiewicz University, Umultowska 89, 61-614 Poznań, Poland
Seppänen et al. 2007; Szymkowiak et al. 2017) and heterospecific alarm calls provide a wealth of information about predation risk (e.g., Magrath et al. 2015).

Some of the most persuasive results of interspecific information use in the wild has been provided using passerine songbirds, i.e., migratory pied and collared flycatchers (Ficedula hypoleuca and Ficedula albicollis) cueing on resident great tits (Parus major) and blue tits (Cyanistes caeruleus), as a model system. Flycatchers were found to use the presence and density of tits for habitat selection and offspring investment decisions (Forsman et al. 2002, 2008; Kivelä et al. 2014) and gain fitness benefits by such behavior (Forsman et al. 2002, 2007). Moreover, in a series of studies (Seppänen and Forsman 2007; Forsman and Seppänen 2011; Seppänen et al. 2011; Loukola et al. 2013), it has been shown that flycatchers selectively either copy or reject nest site preferences of tits when making their own nest site choices, depending on the perceivable fitness correlate (clutch size) of tit tutors (a process termed selective interspecific information use). This was done using a novel experimental design- 
apparent novel niche experiment-in which an apparent preference of tits towards a geometric symbol is simulated by adding a symbol to nest boxes occupied by tits, and an alternative symbol to empty nest boxes. Flycatchers may then either copy or reject tit choice while settling in the neighborhood where the two alternative nest box types are present (Seppänen and Forsman 2007; Forsman and Seppänen 2011; Seppänen et al. 2011; Loukola et al. 2013). The symbols are abstract, evolutionary neutral, and ecologically meaningless, which controls for any innate or learned preference of flycatchers. A series of apparent novel niche experiment studies has shown that when selecting own nest sites, flycatchers copy tit choice if tit clutch size is high, but reject it and prefer an opposite symbol if tit clutch size is low (Seppänen and Forsman 2007; Forsman and Seppänen 2011; Seppänen et al. 2011; Loukola et al. 2013). The apparent novel niche experiment approach has opened new pathways to study social information use in natural settings and the results provided by this approach became textbook examples of interspecific information use in wild animals (e.g., Hoppit and Laland 2013; Stevens 2013; Whitehead and Rendell 2014).

However, the interpretation of results from the apparent novel niche experiment studies has recently been challenged. In particular, Slagsvold and Wiebe (2017) argued that the selective interspecific information use hypothesis is based on several assumptions that have never been examined in the field or are not supported by the available data. Specifically, the authors (i) emphasized the lack of evidence that flycatchers visit tit nests to collect information about clutch size, (ii) questioned the ability of flycatchers to assess tit clutch size, and (iii) argued that the geometric symbols used in the apparent novel niche experiment approach are an uninformative cue and flycatchers would gain no fitness benefits by copying these symbols when choosing their own nest sites. Ultimately, Slagsvold and Wiebe (2017) called to reject the selective interspecific information use hypothesis and proposed the owner aggression hypothesis as an alternative explanation of the earlier results.

According to the owner aggression hypothesis, tits defend extra cavities within their territories and especially these that appear different to the one they currently occupy. Extra cavities are defended for future re-nesting attempts if the current nest is depredated, and their different external appearance is assumed to reduce predation risk from predators forming search images during foraging (Slagsvold and Wiebe 2017). Thus, under the owner aggression hypothesis, patterns of flycatcher nest site choices found in earlier experiments resulted from aggressive interactions, not information use, between tits and flycatchers, i.e., tits preventing flycatchers to settle in neighboring boxes and especially these with a different symbol than on their own box (Slagsvold and Wiebe 2017). Tits with larger clutches are assumed to be more motivated to defend extra cavities (e.g., due to covariance between clutch size and body condition or individual experience), which in turn results in flycatchers apparently copying nest site preferences of tits depending on tit clutch size (Slagsvold and Wiebe 2017).

Several arguments raised against the selective interspecific information use hypothesis have already been addressed and it is clear that for now favoring the owner aggression hypothesis over the selective interspecific information use hypothesis would be premature (Forsman et al. 2018; Samplonius 2018). However, there are points that still remain open in the ongoing debate, related to flycatcher prospecting behavior and cognitive abilities to assess tit clutch size, which call for urgent research attention. Here, I focus on these points of disagreement. Before elaborating on each specific point, I start by briefly summarizing the main arguments that have been already made on it.

\section{Prospecting for interspecific information in flycatchers-the need to consider the environmental context}

Much of the current debate has been devoted to one of the key assumptions of the selective interspecific information use hypothesis, i.e., that flycatchers visit tit nests prior to choosing their own nest site and collect information about tit clutch size. Initially, Slagsvold and Wiebe (2017) argued that there is little evidence for such visits and, based on data about mortality of flycatchers found in tit nests, emphasized that tit nests are visited mainly by males, while if aimed to collect social cues, prospecting could be expected to be female-biased, because females build the nest. However, Forsman et al. (2018) provided new data from a 2-year video monitoring of tit nests and demonstrated that they are frequently visited by flycatchers. Forsman et al. (2018) also argued that male-biased prospecting may result from the stronger effect of a male on nest site choice than previously thought, as repeatedly suggested by recent studies (Kivelä et al. 2014; Morinay et al. 2018). Moreover, in flycatchers, males are known to collect information at the fine-grained local scale, while females prospect at a larger scale (Doligez et al. 2004). Nevertheless, Forsman et al. (2018) found that flycatchers visited tit nests mainly during the egg-laying period of tits and rarely during the incubation period. This may seem in contradiction with the selective interspecific information use hypothesis, because clutch size information would be most reliable during the incubation stage, when clutches are complete. Consequently, Slagsvold and Wiebe (2018) argued that the fundamental proximate mechanism of the selective interspecific information use hypothesis fails, so the hypothesis should be rejected. However, below I argue that interannual variation in breeding phenology of the two species may be key in explaining this 
apparent discrepancy between the timing of information gathering and information value.

In any system involving information use between migrant and resident species, interspecific differences in phenology will be a crucial factor shaping the availability and value of social cues. Under the selective interspecific information use scenario, this factor will be the extent of phenological mismatch between the timing of breeding for tits and the arrival of flycatchers from wintering grounds (Seppänen et al. 2007; Parejo 2016; Forsman et al. 2018). Residents can track changes in environmental conditions at breeding grounds and flexibly adjust their breeding phenology (e.g., Wesołowski et al. 2016). On the contrary, migrants are more constrained in adjusting their seasonal activities and show less flexible phenological shifts than residents (e.g., Both and Visser 2001; Halupka and Halupka 2017). Thus, the extent of phenological mismatch between the timing of tit breeding and flycatcher arrival is expected to show between-year differences, driven mainly by plasticity of tit breeding phenology in response to environmental factors. For example, on Gotland, the time interval between average tit and flycatcher laying date can vary among years from $\sim 8$ days to $\sim 3$ weeks (Morinay et al. 2018).

Flycatchers have a limited time for decision-making upon arrival, as individuals compete for high-quality nest sites and their reproductive success declines with date of breeding (Lundberg and Alatalo 1992). Thus, flycatchers should have no advantage in delaying prospecting until tit egg laying is completed, but instead prospect shortly after arrival. Varying extent of phenological mismatch implies then that in some years flycatchers will prospect tit nests during incubation stage, while in others during egg laying. For flycatchers using tit clutch size as a cue for tit nest site preference, this in turn will affect the availability and value of social information (Parejo 2016). In some years, the information about tit clutch size will be available and reliably reflect final tit investment, but this cue will be less reliable in seasons with higher temporal overlap between the onset of tit breeding and the timing of flycatcher arrival, which in turn may affect social information use patterns. For example, in another system, zebra finches (Taeniopygia guttata) have been found to cue on the breeding success of conspecifics in their own nest site choices, but only when this information was available (Mariette and Griffith 2012). Recently, Morinay et al. (2018) provided support for an analogous mechanism in flycatcher-tit system. Using the apparent novel niche experiment approach, the authors found that flycatchers copied apparent preference of tits in 2012, but rejected it in 2013 (Morinay et al. 2018). This likely resulted from the delayed reproduction of tits in 2013, leading to high temporal overlap between tit reproduction and flycatcher arrival. Consequently, when prospecting upon arrival in 2013, flycatchers detected mainly small tit clutches and rejected tit preferences. Such interannual changes in the availability and value of social information, in turn shaping patterns of decision-making, will emerge in any system where social cues are flexibly adjusted in response to environmental factors (Parejo 2016).

The timing of flycatcher prospecting may also result from the constraints on, and the cost-benefit balance of, accessing tit nests. Inspection of tit nests may be risky for flycatchers, as they may even be killed by nest owners (Forsman et al. 2018; Samplonius and Both 2019). Although flycatchers could probably reduce the mortality risk by determining tit nest box occupation and stage from outside the box, based on the intra-pair vocal activity of tits (Baucaud et al. 2016), assessing tit clutch size would require entering the nest. The access to tit nests will be reduced and riskier during the incubation stage, when female tits spend more time in the nest than during egg laying (Slagsvold and Wiebe 2017). Consequently, flycatchers prospecting during tits egg laying-especially when almost complete clutches are laid-may result from a trade-off between the value, availability, and cost of acquisition of social information.

So far, the apparent novel niche experiment studies have revealed how flycatchers use interspecific information if this information is available. However, we still have little understanding of how flycatchers gather social cues from tits. Future research efforts should focus on comprehensive studies on prospecting behavior in flycatcher-tit system using radio frequency identification (RFID) technology and passive integrated transponder (PIT) tags (Bonter and Bridge 2011; Ponchon et al. 2013). Application of RFID will allow creating networks of remotely monitored nest boxes, each equipped with a loop antenna mounted around the entrance hole and a data logger (Moks et al. 2016; Schuett et al. 2017). In turn, this will allow automated tracking of within-nest prospecting visits made by individuals equipped with PIT tags throughout the breeding season. Tracking prospecting behavior should span multiple seasons and be coupled with standard population monitoring, to account for interannual variation in breeding synchrony between tits and flycatchers. Prospecting behavior could also be studied experimentally, e.g., by enlarging or reducing tit clutch size (Schuett et al. 2017), or even advancing or delaying tit breeding phenology (Samplonius and Both 2017), and investigating how such manipulations affect the patterns of flycatchers prospecting behavior. Such studies will provide broadly valuable insights on mechanisms of early prospecting and the role of environmental factors in shaping patterns of social information use in migrant-resident systems.

\section{Assessment of tit clutch size}

Several points of disagreement in the ongoing debate emerged around cognitive abilities of flycatchers to assess tit clutch size. In particular, Slagsvold and Wiebe (2017, 2018) 
questioned whether flycatchers can "count" eggs in tit nests and this process was suggested to be very difficult because of the small variation in tit clutch size. Forsman et al. (2018) and Samplonius (2018) have already pointed to the many examples of numerical abilities in birds, although acknowledging that the mechanism of tit clutch size assessment by flycatchers remains to be shown. Numerical cognition has ancient evolutionary roots and the phylogenetic ubiquity of at least basic sense of numbers throughout the animal kingdom is now widely acknowledged, with some bird species being among the most renowned for their quantitative skills (Shettleworth 2010; Butterworth et al. 2017; Nieder 2017). In addition, I argue that there is no reason to assume that distinguishing between small and large tit clutches by flycatchers is a numerical distinction at all, or that this process requires very high accuracy for selective social information use to evolve.

Quantity judgements play a fundamental role in everyday life of most organisms, which have very different cognitive abilities. Animals, from insects to mammals, routinely discriminate between small and large quantity or size in a variety of contexts. For example, female tungara frogs (Physalaemus pustulosis) compare males based on the number of advertising calls they produce (Rose 2017). Similarly, male wood warblers (Phylloscopus sibilatrix) adjust territory defense behavior depending on the number of songs sung by an intruder (Szymkowiak and Kuczyński 2017). Quantity judgements may be also an important aspect of predator avoidance strategies in prey species, e.g., guppies (Poecilia reticulata) preferentially join larger shoals to reduce predation risk (Agrillo and Bisazza 2017). The ability to use quantitative information is innate, does not reflect any higher cortical functions, occurs in a variety of ecological contexts, and has crucial fitness effects (Shettleworth 2010; Butterworth et al. 2017). Thus, there is no reason to expect the assessment of tit clutch size by flycatchers to differ from the previous examples, nor that this process involves "counting" tit eggs or requires any sophisticated cognitive capacities.

Discriminating high vs. low tit clutch size was suggested to be a challenging task for flycatchers because of the low variation in clutch sizes at the local scale (Slagsvold and Wiebe 2017). In fact, one of the studies using apparent novel niche experiment approach (Seppänen et al. 2011) showed that flycatchers do not discriminate between clutches of similar size (e.g., 6 vs. 7 eggs). Nevertheless, tit clutch size shows considerable variation and even at the local scale may range from 1 to 16 eggs (Perrins 1965). Moreover, the very high accuracy of clutch size assessment in flycatchers should not be expected, nor is it required for the emergence of selective social information use. The information conveyed by clutches of close size is very similar. Thus, fitness consequences of discriminating between clutches differing by 1 or 2 eggs are a lot smaller than discriminating between clutches considerably differing in size (e.g., 4 vs. 13 eggs as in apparent novel niche experiment). Consequently, the lack of flycatcher ability to discriminate between tit clutches of similar size does not preclude the evolution of selective interspecific social information use.

Further points of disagreement in the debate referred to the mechanistic aspects of tit clutch size assessment by flycatchers. Slagsvold and Wiebe $(2017,2018)$ argued that tactile stimulation of a brood patch is unlikely to be involved in assessing tit clutch size, since prospecting in flycatchers is male-biased and males lack a brood patch. In addition, cavities were suggested to be too dark to allow visual assessment, as proposed by Forsman et al. (2018). The exact mechanism of clutch size assessment in flycatchers remains to be unraveled, but the hypothesis that flycatchers rely on visual cues when assessing tit clutch size should not be abandoned, nor should it be assumed a sensory challenge. Flycatcher decision-making is known to be influenced by the visibility of tit eggs (Loukola et al. 2013) and tits have been experimentally shown to increase egg covering in simulated presence of flycatchers, with one function of such behavior could be a counter-adaptation against information gathering by flycatchers (see Loukola et al. 2014 for detailed discussion). This points towards the importance of flycatchers actually seeing tit clutch when using it as a cue for nest site selection. Moreover, natural cavities occupied by tits are dark, but the range of illuminance values in natural cavities occupied by flycatchers $(0.01-0.41 \mathrm{x}$; Maziarz and Wesolowski 2014) is within the lower range of illuminance recorded in tit cavities $(0.03-3 \mathrm{~lx}$; Wesołowski and Maziarz 2012). Thus, flycatcher cavities are usually even darker than tit cavities, suggesting that flycatchers might be adapted to see in dim light. If being a sensory challenge at all, varying light conditions encountered by prospecting flycatchers (e.g., due to variation in deepness of tit cavities) will rather be just another environmental factor shaping the availability of information.

Detailed studies on the mechanisms of tit clutch size assessment by flycatchers may open a fruitful area of research on proximate aspects of social information gathering in birds. Discriminating clutch size assessment via tactile stimulation from visual cues would be technically difficult. However, whether flycatchers rely on visual cues can be studied within the apparent novel niche experiment approach, using dummy tit nests (e.g., Forsman et al. 2012) and with experimental manipulation of egg visibility by covering the eggs, e.g., with lining materials. Moreover, using cryptic eggs painted with a color matching the nesting material and placed among nonpainted visible eggs would allow providing conflicting visual and tactile information about tit clutch size for flycatchers. Furthermore, effects of light conditions within tit cavities on information gathering by flycatchers from tits can also be studied using a range of nest box types with different depth (and thus illuminance; Wesołowski and Maziarz 2012; Maziarz and Wesołowski 2014) or nest boxes equipped with 
transparent, plexiglass windows located in side-walls along with adjustable shutters to manipulate light conditions within the box (Podkowa and Surmacki 2017).

\section{Final thoughts}

For now, there is no unequivocal evidence against the selective interspecific information use hypothesis and it still appears a more parsimonious explanation of results from earlier studies than the owner aggression hypothesis (Forsman et al. 2018; Samplonius 2018). However, the ongoing debate highlighted several gaps in the mechanistic understanding of information gathering between flycatchers and tits (Slagsvold and Wiebe 2017, 2018). Addressing them will allow to ultimately resolve the selective interspecific information use vs. owner aggression hypothesis dilemma and expand our general understanding of the proximate basis of using social cues for nest site selection in wild birds. This current debate also brings about one broadly applicable lesson for animal behavior research. When focusing mostly on proximate mechanisms, there is a risk of incorrectly assuming complex cognitive requirements for tasks that animals across taxa perform routinely and that are vital for fitness, as for example quantity judgements. Instead, considering the evolutionary significance of behavior, rather than exclusively focusing on detailed mechanisms, will provide a more powerful approach when attempting to invalidate competing hypotheses about behavioral adaptations in animals.

Acknowledgements I thank Jukka T. Forsman and Robert L. Thomson for all discussions about social information use in flycatchers and tits, as well as comments on the earlier versions of the manuscript. I also thank the Associate Editor, Blandine Doligez, and an anonymous reviewer for valuable feedback upon earlier drafts of this text. I am supported by the Foundation for Polish Science (FNP) scholarship "Start."

Open Access This article is distributed under the terms of the Creative Commons Attribution 4.0 International License (http:// creativecommons.org/licenses/by/4.0/), which permits unrestricted use, distribution, and reproduction in any medium, provided you give appropriate credit to the original author(s) and the source, provide a link to the Creative Commons license, and indicate if changes were made.

\section{References}

Agrillo C, Bisazza A (2017) Understanding the origin of number sense: a review of fish studies. Phil Trans R Soc B 373:20160511

Avarguès-Weber A, Dawson EH, Chittka L (2013) Mechanisms of social learning across species boundaries. J Zool 290:1-11

Baucaud ICA, Valère PA, Aquirre Smith MLN, Doligez B, Cauchard L, Rybak F, Vignal C (2016) Interactive vocal communication at the nest by parent great tits Parus major. Ibis 158:630-644
Bonter DN, Bridge ES (2011) Applications of radio frequency identification (RFID) in ornithological research: a review. J Field Ornithol 82:1-10

Both C, Visser ME (2001) Adjustment to climate change is constrained by arrival date in a long-distance migrant bird. Nature 411:296-298

Butterworth B, Gallistel CR, Vallortigara G (2017) Introduction: the origins of numerical abilities. Phil Trans R Soc B 373:20160507

Doligez B, Pärt T, Danchin E (2004) Prospecting in the collared flycatcher: gathering public information for future breeding habitat selection? Anim Behav 67:457-466

Forsman JT, Seppänen J-T (2011) Learning what (not) to do: testing rejection and copying of simulated heterospecific behavioural traits. Anim Behav 81:879-883

Forsman JT, Seppänen J-T, Mönkkönen M (2002) Positive fitness consequences of interspecific interaction with a potential competitor. Proc R Soc Lond B 269:1619-1623

Forsman JT, Thomson RL, Seppänen J-T (2007) Mechanisms and fitness effects of interspecific information use between migrant and resident birds. Behav Ecol 18:888-894

Forsman JT, Hjernquist MB, Taipale J, Gustafsson L (2008) Competitor density cues for habitat quality facilitating habitat selection and investment decisions. Behav Ecol 19:539-545

Forsman JT, Seppänen J-T, Nykänen IL (2012) Observed heterospecific clutch size can affect offspring investment decisions. Biol Lett 8: 341-343

Forsman JT, Seppänen J-T, Mönkkönen M, Thomson RL, Kivelä SM, Krams I, Loukola OJ (2018) Is it interspecific information use of aggression between putative competitors that steers the selection of nest-site characteristics? A reply to Slagsvold and Wiebe. J Avian Biol 49:e01558

Halupka L, Halupka K (2017) The effect of climate change on the duration of avian breeding seasons: a meta-analysis. Proc R Soc B 284: 20171710

Hoppit W, Laland K (2013) Social learning: an introduction to mechanisms, methods, and models. Princeton Univ Press, Princeton

Kivelä SM, Seppänen J-T, Ovaskainen O, Doligez B, Gustafsson L, Mönkkönen M, Forsman JT (2014) The past and the present in decision-making: the use of conspecific and heterospecific cues in nest site selection. Ecology 95:3428-3439

Loukola OJ, Seppänen J-T, Krams I, Torvinen SS, Forsman JT (2013) Observed fitness may affect niche overlap in competing species via selective social information use. Am Nat 182:474-483

Loukola OJ, Laaksonen T, Seppänen J-T, Forsman JT (2014) Active hiding of social information from information parasites. BMC Evol Biol 14:32

Lundberg A, Alatalo RV (1992) The pied flycatcher. Poyser, London

Magrath RD, Haff TM, Fallow PM, Radford AN (2015) Eavesdropping on heterospecific alarm calls: from mechanisms to consequences. Biol Rev 90:560-586

Mariette MM, Griffith SC (2012) Conspecific attraction and nest site selection in a nomadic species, the zebra finch. Oikos 121:823-834

Maziarz M, Wesołowski T (2014) Does darkness limit the use of tree cavities for nesting by birds? J Ornitho 155:793-799

Moks K, Tilgar V, Thomson RL, Calhim S, Järvisto PE, Schuett W, Velmala W, Laaksonen T (2016) Predator encounters have spatially extensive impacts on parental behaviour in a breeding bird community. Proc R Soc B 283:20160020

Morinay J, Forsman JT, Kivelä SM, Gustafsson L, Doligez B (2018) Heterospecific nest site copying behavior in a wild bird: assessing the influence of genetics and past experience on a joint breeding phenotype. Front Ecol Evol 5. https://doi.org/10.3389/fevo.2017. 00167

Nieder A (2017) Evolution of cognitive and neural solutions enabling numerosity judgements: lessons from primates and corvids. Phil Trans R Soc B 373:20160514 
Parejo D (2016) Information mismatches: a neglected threat of climate change to interspecific interactions. Front Ecol Evol. 4. https://doi. org/10.3389/fevo.2016.00031

Perrins CM (1965) Population fluctuations and clutch-size in the great tit, Parus major L. J Anim Ecol 34:601-647

Podkowa P, Surmacki A (2017) The importance of illumination in nest site choice and characteristics of cavity nesting birds. Sci Rep 7: 1329

Ponchon A, Grémillet D, Doligez B, Chambert T, Tveraa T, GonzálezSolís J, Boulinier T (2013) Tracking prospecting movements involved in breeding habitat selection: insights, pitfalls and perspectives. Methods Ecol Evol 4:143-150

Rose GJ (2017) The numerical abilities of anurans and their neural correlates: insights from neuroethological studies of acoustic communication. Phil Trans R Sco B 373:20160512

Samplonius JM (2018) Does territory owner aggression offer an alternative explanation to patterns in heterospecific information use studies? A comment on Slagsvold \& Wiebe. J Avian Biol 49:e01567

Samplonius JM, Both C (2017) Competitor phenology as a social cue in breeding site selection. J Anim Ecol 86:615-623

Samplonius JM, Both C (2019) Climate change may affect fatal competition between two bird species. Curr Biol 29:327-331

Schuett W, Järvisto PE, Calhim S, Velmala W, Laaksonen T (2017) Nosy neighbours: large broods attract more visitors. A field experiment in the pied flycatcher, Ficedula hypoleuca. Oecologia 184:115-126

Seppänen J-T, Forsman JT (2007) Interspecific social learning: novel preference can be acquired from a competing species. Curr Biol $17: 1-5$

Seppänen J-T, Forsman JT, Mönkkönen M, Thomson RL (2007) Social information use is a process across time, space, and ecology, reaching heterospecifics. Ecology 88:1622-1633

Seppänen J-T, Forsman JT, Mönkkönen M, Krams I, Salmi T (2011) New behavioural trait adopted or rejected by observing heterospecific tutor fitness. Proc R Soc Lond B 278:1736-1741
Shettleworth SJ (2010) Cognition, evolution, and behavior. Oxford Univ Press, Oxford

Slagsvold T, Wiebe KL (2017) On the use of heterospecific information for nest site selection in birds. J Avian Biol 48:1035-1040

Slagsvold T, Wiebe KL (2018) On heterospecific learning in birdscomments on Samplonius and Forsman et al. J Avian Biol 49: e01706

Stevens M (2013) Sensory ecology, behaviour, and evolution. Oxford Univ Press, Oxford

Szymkowiak J, Kuczyński L (2017) Song rate as a signal of male aggressiveness during territorial contests in the wood warbler. J Avian Biol 48:275-283

Szymkowiak J, Thomson RL, Kuczyński L (2017) Interspecific social information use in habitat selection decisions among migrant songbirds. Behav Ecol 28:767-775

Thomson RL, Forsman JT, Mönkkönen M (2003) Positive interactions between migrant and resident birds: testing the heterospecific attraction hypothesis. Oecologia 134:431-438

Wesołowski T, Maziarz M (2012) Dark tree cavities - a challenge for hole nesting birds? J Avian Biol 43:454-460

Wesołowski T, Cholewa M, Hebda G, Maziarz M, Rowiński P (2016) Immense plasticity of timing of breeding in a sedentary forest passerine, Poecile palustris. J Avian Biol 47:129-133

Whitehead H, Rendell L (2014) The cultural lives of whales and dolphins. Univ Chicago Press, Chicago

Publisher's note Springer Nature remains neutral with regard to jurisdictional claims in published maps and institutional affiliations. 